

\section{DISCLAIMER}

Portions of this document may be illegible in electronic image products. Images are produced from the best available original document. 


\title{
Lifetime Evaluation of Plasma-Facing Materials during A Tokamak Disruption*
}

\author{
A. Hassanein ${ }^{a}$ and I. Konkashbaevb \\ aArgonne National Laboratory, Argonne, IL, USA \\ b Troitsk Institute for Innovation, Troitsk, Russian Federation
}

\begin{abstract}
Erosion losses of plasma-facing materials in a tokamak reactor during major disruptions, giant ELMs, and large power excursions are serious concerns that influence component survivability and overall lifetime. Two different mechanisms lead to material erosion during these events: surface vaporization and loss of the melt layer. Hydrodynamics and radiation transport in the rapidly developed vapor-cloud region above the exposed area are found to control and determine the net erosion thickness from surface vaporization. A comprehensive selfconsistent kinetic model has been developed in which the time-dependent optical properties and the radiation field of the vapor cloud are calculated in order to correctly estimate the radiation flux at the divertor surface. The developed melt layer of metallic divertor materials will, however, be free to move and can be eroded away due to various forces. Physical mechanisms that affect surface vaporization and cause melt layer erosion are integrated in a comprehensive model. It is found that for metallic components such as beryllium and tungsten, lifetime due to these abnormal events will be controlled and dominated by the evolution and hydrodynamics of the melt layer during the disruption. The dependence of divertor plate lifetime on various aspects of plasma/material interaction physics is discussed.
\end{abstract}

*Work supported by the U. S. Department of Energy, Office of Fusion Energy. 


\section{Introduction}

The high energy deposited during abnormal events such as major disruptions, giant edge-localized modes (ELMs), and large power excursions on plasma-facing materials (PFMs) such as the divertor plate will lead to very high surface temperatures that will cause melting and vaporization. As a result, erosion of plasma-facing materials during these events is viewed as a critical obstacle to reliable and successful operation of a tokamak reactor. Two separate mechanisms lead to material erosion, and consequently to shorter component lifetime, during such abnormal events: surface vaporization and loss of the developed melt layer of metallic components. The initial burst of energy deposited will cause a sudden vapor cloud to form above the exposed area, significantly reducing the net energy flux to the divertor plate and leading to a substantial reduction of further material erosion. To analyze these issues, the detailed physics of plasma/solid-liquid/vapor interactions in a strong and oblique magnetic field must be developed and evaluated in a self-consistent manner.

The magnetohydrodynamics and radiation transport in the vapor-cloud region are found to control and determine the net erosion thickness from surface vaporization. In this analysis, magnetic field diffusion in the vapor, twodimensional effects of vapor expansion, and lateral radiation losses are taken into account. However, because of the shielding-layer effect, the thickness of the developed melt-layer is much larger than the vaporized thickness. During a disruption, the melt layer will be free to move, subject to various forces such as electromagnetic, gravitation, mechanical vibration, plasma particle momentum ("plasma-wind"), ablation recoil, and surface tension. Some of these forces can also trigger certain hydrodynamic instabilities, which in turn may cause melt-layer 
losses. Various mechanisms responsible for melt-layer erosion are discussed. It is concluded that for metallic plasma-facing materials, such as beryllium and tungsten, lifetimes due to these abnormal events will be controlled and dominated by the hydrodynamics of the melt layer and the development and growth of instabilities. Because ITER-like heat loads and disruption conditions are not achievable in current tokamak machines, laboratory experiments are used to study and simulate disruption effects. Modeling of these experiments and their relevance to reactor conditions during a disruption are discussed. The overall dependence of divertor-plate lifetime on various aspects of plasma/ material interaction physics is analyzed and discussed.

\section{Comprehensive self-consistent model}

The comprehensive model developed in the A*THERMAL-S code has been extensively used to evaluate PFMs response to disruptions [1-2]. In this model, three major modeling stages of plasma/material interaction were developed with sufficient detail to accurately simulate disruption effects. Initially, the incident plasma particles from the disrupted plasma will deposit part of their energy on the PFC surface. Models for particle deposition and material thermal evolution that take into account phase change, moving boundaries, and temperature-dependent thermophysical properties, etc., were developed to predict the behavior of these components. This initial burst of energy delivered to PFM surfaces from the direct impact of plasma particles will cause sudden ablation of these materials. As a result, a vapor cloud will form in front of the incoming plasma particles. Shortly thereafter, the plasma particles will be completely stopped in this vapor cloud. Continuous heating of the vapor cloud 
will ionize, excite, and generate photon radiation. The kinetic energy of the incoming plasma particle is therefore transformed into radiation energy.

Comprehensive models for the hydrodynamics and heating of the vapor cloud that shields the original surface were developed for the second stage of disruption modeling. Finally, models for radiation transport throughout the vapor cloud were developed to estimate the net heat flux transmitted to the facing materials. Because of the importance of radiation transport in the vapor cloud region, a self-consistent approach [3] to calculate the actual radiation field is also developed and implemented in the A*THERMAL-S code. The optical properties of the vapor-cloud plasma are calculated at each time-step during the course of the disruption. The relevant atomic data bases of both carbon and beryllium are implemented in the code and include all possible transition energy levels, ionization potentials, rate coefficients, photoionization cross sections, statistical probabilities, oscillator strengths, etc. The rate equations are then solved for each level population at each time step. The radiation transport equation is then solved separately for both line- and continuum-generated spectra. The selfconsistent model also takes into account the multispecies effect, i.e., mixing between the incoming plasma particles and the vaporized material. This turns out to be critically important in explaining, for the first time, fine details of disruption simulation experiments.

Detailed effects of the in-reactor strong magnetic field on vapor hydrodynamics and subsequent effects of both low- and high-atomic number materials were examined. Figure 1 is a schematic illustration of the interaction of plasma particles with the divertor plate in a strong magnetic field environment. Coordinate $X$ is the toroidal direction, $Z$ is the poloidal direction, and $Y$ is the 
normal direction above the divertor plate. The model also includes effects such as 2-D vapor expansion along and parallel to field lines, magnetic field diffusion in the vapor cloud, magnetic and friction forces, and Joule heating of the vapor [2]. The reactor magnetic field is found to limit vapor expansion normal to the divertor surface. This is particularly important for the case of a closed divertor configuration. The expanding hot vapor plasma can significantly damage other reactor components that are not directly exposed to plasma disruption. It is therefore the dynamics and evolution of this vapor cloud that will finally determine the net erosion rate at the end of a disruption.

\section{Results}

In this analysis, thermal disruption quench times of $0.1-1.0 \mathrm{~ms}$ and plasma energy densities of $10-100 \mathrm{MJ} / \mathrm{m}^{2}$ are used to evaluate ITER disruption conditions. Other disruption times and energy densities can also be easily examined with the codes used here. Candidate PFMs such as beryllium, tungsten, and carbon were considered in this analysis.

Figure 2 shows the tungsten and beryllium solid/liquid/vapor temperatures as a function of distance during a disruption of $10 \mathrm{MJ} / \mathrm{m}^{2}$ energy density and 0.1 ms deposition time. Because $W$ is a refractory metal, its surface and bulk temperatures can be much higher than those of $\mathrm{Be}$ under the same disruption conditions. Tungsten melt-layer thickness is about twice that of Be. The $\mathrm{Be}$ vapor temperature is, however, much higher than the $W$ vapor. This is because of the higher $W$ atomic number, which increases vapor radiation and therefore cooling of the vapor. 
Most of the disrupting plasma energy flux is converted into radiation energy from the hot vapor cloud during the early stages of the disruption. Therefore, a detailed radiation transport calculation is required to estimate the net radiated energy flux for all vapor regions back to the divertor surface. In addition to the time-dependent self-consistent model used to calculate the local radiation field at each vapor zone, the code can also use multigroup opacity and emissivity precalculated data to conserve computer time [1]. Line radiation in the vapor is particularly important for low-atomic number materials and high-temperature vapor clouds. Line radiation and its transport are calculated separately and then added to the continuum radiation for accurate evaluation of net heat load at the divertor surface.

Figure 3 shows the calculated photon spectrum for the outermost vapor Fig. 3 regions of both $\mathrm{Be}$ and $\mathrm{W}$ vapor. In most $\mathrm{Be}$ vapor disruption conditions, more than $90 \%$ of the hot-region radiation is attributed to line radiation. One concern is that the hot vapor may expand far above the divertor surface and cause damage to other components that are not directly exposed to the disrupting plasma. Figure 4 shows $B e$ and $W$ vapor-expansion velocity components in the $X$ direction (along the divertor surface) and in $Y$ direction (normal to surface) under the effect of a 5-T inclined magnetic field. Initially, $V_{y}$ is higher than $V_{x}$ because the ablated material leaves the surface as neutral atoms in the normal direction, but is then ionized and begins to follow the magnetic field lines. It can be seen from Figs. 2 and 4 that light elements $(\mathrm{Be})$ and heavy elements $(W)$ have similar expansion distances, but for different reasons. For $W$, the vapor temperature is low $(<5 \mathrm{eV})$, which means low vapor electric conductivity, $\sigma$, and consequently a high diffusion coefficient of the vapor. Therefore, $W$ vapor diffuses freely across magnetic field lines. This expansion velocity is calculated to be 
$V_{\perp} \approx 0.2-0.4 V_{T}$ (thermal velocity). However, because of the high atomic number of $W$ and its lower temperature, its thermal velocity $V_{T} \approx 1-2 \mathrm{~km} / \mathrm{s}$. Vapor expansion of $W$ in the vertical direction is then limited to less than $10 \mathrm{~cm}$ at the end of disruption. For light elements $(\mathrm{Be})$, the vapor normal expansion is limited by the much lower diffusion across magnetic field lines, due to the higher vapor temperatures.

The energy density deposited on the divertor surface during a disruption can exceed $100 \mathrm{MJ} / \mathrm{m}^{2}$. Figure 5 shows $\mathrm{W}$ solid/liquid/vapor temperatures as a Fig. 5 function of distance for $100 \mathrm{MJ} / \mathrm{m}^{2}$ energy density deposited at two different disruption times, i.e., $0.1 \mathrm{~ms}$ and $1 \mathrm{~ms}$. At the shorter disruption, both the liquid/ solid and the vapor temperatures are higher than at the longer disruption. A longer disruption time causes the vapor to expand to greater distances above the divertor surface and also causes the energy flux at the divertor surface to diffuse deeper into the bulk and produces a thicker melt layer. Depending on divertor design and configuration, the expanding hot vapor and its radiation can damage nearby components, particularly in closed divertor configurations. It is therefore desirable to keep vapor normal expansion to a minimum.

The net erosion rate from surface vaporization of $\mathrm{C}, \mathrm{Be}$, and $\mathrm{W}$ candidate Fig. 6 materials exposed to similar disruption conditions is shown in Fig. 6. The carbon vaporization rate is higher than metallic beryllium and tungsten, as determined by the complex interplay of material properties and thermodynamics, vapor magnetohydrodynamics, and detailed photon radiation transport. These interaction processes must be linked dynamically in a self-consistent way to correctly evaluate divertor plate erosion rates. 


\section{Melt-layer erosion}

Because of the large optical thickness of the vapor cloud generated near the divertor surface during the disruption, only a small fraction $(<10 \%)$ of the radiated energy flux reaches the material surface. The erosion thickness of candidate materials from vaporization is generally less than $10 \mu \mathrm{m}$ over a wide range of incident particle flux. The melt-layer thickness of metallic candidate materials, however, can be higher by more than one order of magnitude. Figures 7 and 8 show melt layer and vaporization thicknesses at different energy densities and disruption times for Be and W. Beryllium vaporization thickness is higher than that of $\mathrm{W}$, but its melt-layer thickness is lower. Longer disruption times and higher energy densities usually result in thicker melt layers. At a disruption energy density of $100 \mathrm{MJ} / \mathrm{m}^{2}$ and a disruption time of $1 \mathrm{~ms}, \mathrm{~W}$ meltlayer thickness is $>0.2 \mathrm{~mm}$ while that of $B e$ is $<0.1 \mathrm{~mm}$.

During a disruption, the melt layer is subject to various forces such as electromagnetic, gravitation, mechanical vibration, plasma momentum, surface tension, and ablation recoil [4]. Several mechanisms can cause melt-layer loss during the thermal quench phase of the disruption [5]. One mechanism that can contribute to melt-layer loss is melt splashing from overheating of the melt layer due to formation, growth, and boiling of gas bubbles. Another mechanism of melt-layer erosion is splashing due to absorption of plasma momentum. A further important erosion-causing mechanism is instabilities that develop in the liquid layer because of various forces acting on the free surface of the liquid. Models to study melt-layer erosion due to various mechanisms are implemented in the SPLASH computer code [6]. The code is in good agreement with recent disruption simulation experiments on $\mathrm{Be}$ and on $\mathrm{Al}$ targets which have shown, 
from postexperimental evaluation, that much or all of the melted layer had been spiashed and lost $[7,8]$.

\section{Simulation experiments}

In current tokamak machines, ITER-like heat loads and disruption conditions are not achievable. Therefore, the expected ITER conditions during a plasma disruption must be simulated in the laboratory. Experiments using laser light, electron beams, and plasma guns have been used in several countries to study disruption effects on candidate divertor materials. The majority of these experiments have near-reactor-relevant disruption parameters (i.e., heat loads of $10-20 \mathrm{MJ} / \mathrm{m}^{2}$ and deposition times of $<1 \mathrm{~ms}$ ). The erosion results from these simulation experiments however, do not generally agree with each other for various reasons. Laser beam simulation experiments usually produce higher erosion rates, mainly because vapor shielding is less important. Because the laser beam is very small $(\approx 1 \mathrm{~mm})$, it penetrates the freely expanding vaporized material with little attenuation. Erosion from highly energetic electron beams is also high because of the long range of the electrons in both the vapor and the condensed phase materials.

The $A^{*}$ THERMAL-S code has been used to analyze simulation experiments. The results agree well with electron beam simulations and resulting vapor-interferometry data $[9,10]$. The most relevant plasma-gun simulation experiments of ITER disruption are being conducted in the $2 \mathrm{MK}-200$ facility [11], which can produce deuterium plasma in a magnetic field of $2 \mathrm{~T}$, with total energy up to $50 \mathrm{KJ}$ and particle kinetic energy close to $1 \mathrm{keV}$. Power density can be as high as $10 \mathrm{MW} / \mathrm{cm}^{2}$, with a maximum pulse duration, however, of only $20 \mu \mathrm{s}$. 
Because of these limitations, the $2 \mathrm{MK}-200$ is most useful for studying the early stages of a tokamak disruption and the initial characteristics of plasma/vapor interactions. Erosion data obtained from this device are minimal, compared to those expected during ITER disruptions. Optical interferometry was used to determine the spatial distribution of plasma electron density and temperature in the target vapor.

The self-consistent model with multispecies mixture implemented in the $A^{*}$ THERMAL-S code is used to simulate a recent disruption experiment at the 2MK-200 facility. In the first microsecond, a dense carbon vapor cloud $\left(\mathrm{N}_{\mathrm{e}} \approx 5 \mathrm{x}\right.$ $10^{22} \mathrm{~m}^{-3}$ ) formed above the surface, in good agreement with the experimental data [12]. Figure 9 compares calculations and data from the simulation experiments and ITER conditions of the initial vapor cloud density and temperature. Because of the low kinetic energy of the ions in plasma-gun simulation experiments $\left(E_{i} \ll 10-20 \mathrm{keV}\right)$, the density of the incident plasma particles is of the same or higher order than that of vaporized target material. Therefore, it is necessary to account for the influence of plasma particles on vapor hydrodynamics and on radiation transport [3]. Initially, the $\mathrm{D}^{+}$plasma ions deposit their energy in the target material and then in the target vapor. Soon after, the density of the stopped plasma particles becomes comparable and can exceed that of the target vapor. After a few microseconds, an almost pure deuteron plasma particles exist at the front region of the vapor cloud, as shown in Fig. 9. Most of the incoming energy is further deposited and stored as thermal energy in the plasma particles. Part of this energy is transferred to the target vapor behind the mixture region via electron heat conduction. The calculated electron temperature and density are in good agreement with measured data. In ITER, the situation is different. The density of incoming plasma particles is much 
less than that of the target vapor. Only a small fraction of the energy is stored as thermal energy. Most of the deposited energy is radiated from the target vapor to the divertor surface and other components. This may cause more erosion in ITER than expected from gun experiments, for the same initial disruption energy and deposition time.

\section{Conclusions}

Several aspects of plasma disruption and simulation physics have been studied with a comprehensive self-consistent model that integrates and simultaneously links different stages of a disruption. Loss of the melt layer developed during disruption and ELMs can significantly shorten metallic PFMs lifetime and diminish reactor economic feasibility. The self-consistent approach with multispecies mixing is used to calculate the time-dependent optical properties of the vapor cloud. This helps explain, for the first time, recent experimental data. Major differences exist among current disruption simulation experiments and actual tokamak disruption, and care should be taken in interpreting and extrapolating such data to reactor conditions. More-detailed modeling of melt layer loss, e.g., synergistic interaction of various forces, modeling of vapor cloud diffusion away from the incoming plasma, and modeling possible vapor turbulence and instabilities should be analyzed. In addition, morerelevant simulation experiments are required to correctly assess erosion lifetimes during tokamak disruptions and ELMs. 


\section{References}

[1] A. Hassanein and I. Konkashbaev, J. Nucl. Mater. 220-222 (1995) 244.

[2] A. Hassanein and I. Konkashbaev, Fusion Engineering and Design 28 (1995) 27.

[3] A. Hassanein and I. Konkashbaev, Argonne National Laboratory Report ANL/FPP/TM-286, Oct. 1995.

[4] A. Hassanein, Fusion Technology 15 (1989) 513.

[5] A. Hassanein et al., Fusion Technology, Vol. 1, Aug. (1994) 223.

[6] A. Hassanein and I. Konkashbaev, Suppl. J. Nuclear Fusion, Vol. 5 (1994) 193.

[7] V. Litunovsky, Efremov Institute, Russia (1995), private communications.

[8] A. Hassanein et al., J. Nucl. Mater. 212-215 (1994) 1272.

[9] V. Engelko et al., J. Nucl. Mater. 220-222 (1995) 1071.

[10] A. Hassanein, Fusion Technology, Vol. 26, Nov. (1994) 532.

[11] N. Arkhipov et al., Fusion Technology, Vol. 1, Aug. (1994) 463. 
[12] N. Arkhipov et al., Fusion Technology, Vol. 1, Aug. (1994) 395. 


\section{Figure Captions}

Figure 1

Figure 2

Figure 3

Figure 4

Figure 5

Figure 6

Figure 7

Figure 8
Schematic illustration of plasma-target interaction in a strong magnetic field environment.

Target and vapor temperatures as a function of distance for both tungsten and beryllium.

Photon radiation spectra emitted from tungsten and beryllium vapor.

Beryllium and tungsten vapor expansion velocities along $\left(V_{x}\right)$ and normal $\left(V_{y}\right)$ to divertor surface.

Target and vapor temperatures of tungsten at different disruption times.

Vaporization losses during a disruption for different candidate materials.

Beryllium melt-layer and vaporization thicknesses at different energy densities and disruption times.

Tungsten melt-layer and vaporization thicknesses at different energy densities and disruption times. 
Figure 9

Initial vapor-cloud density and temperature for both ITER and 2MK-200 facility. 


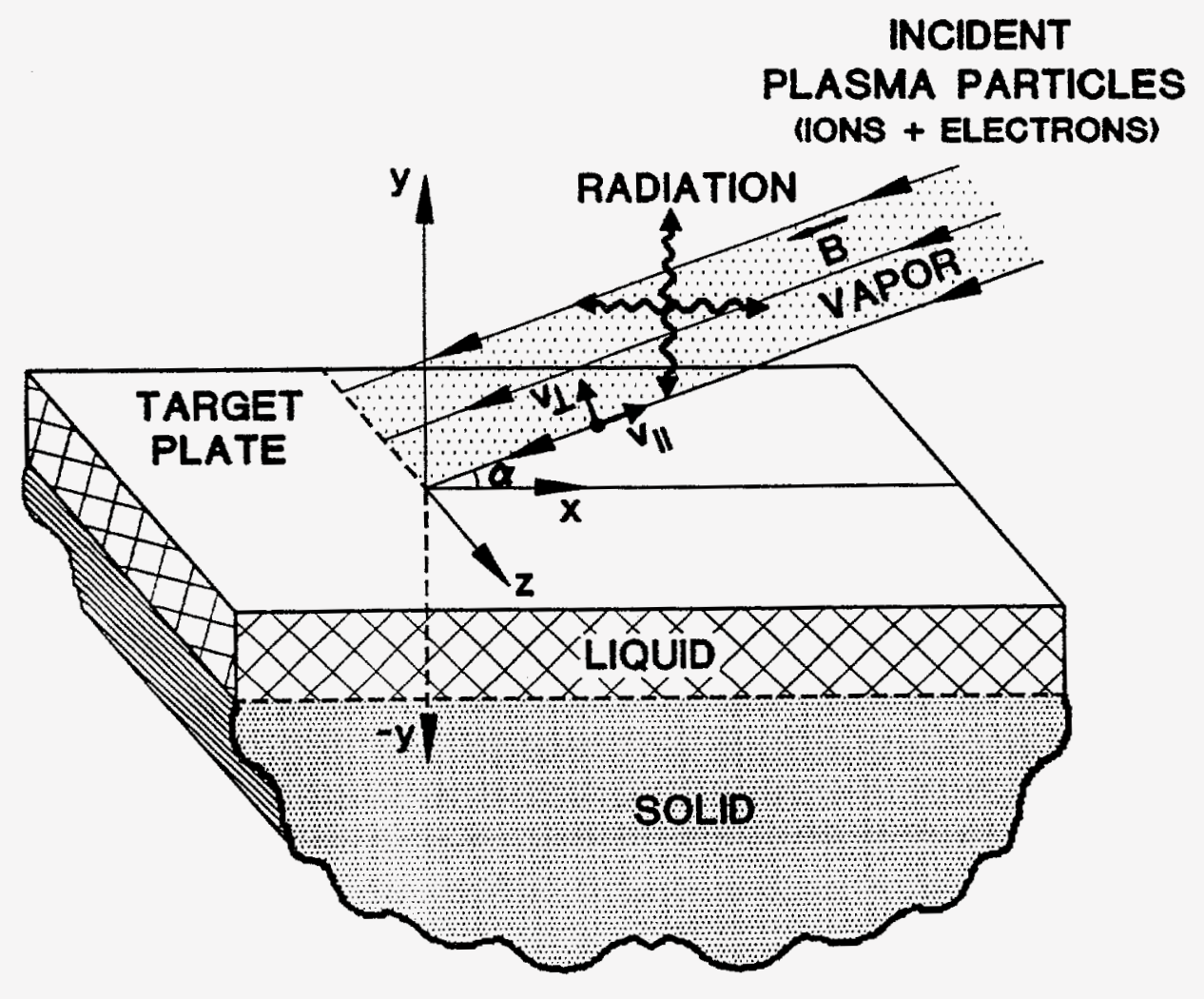

Fig. (1) 


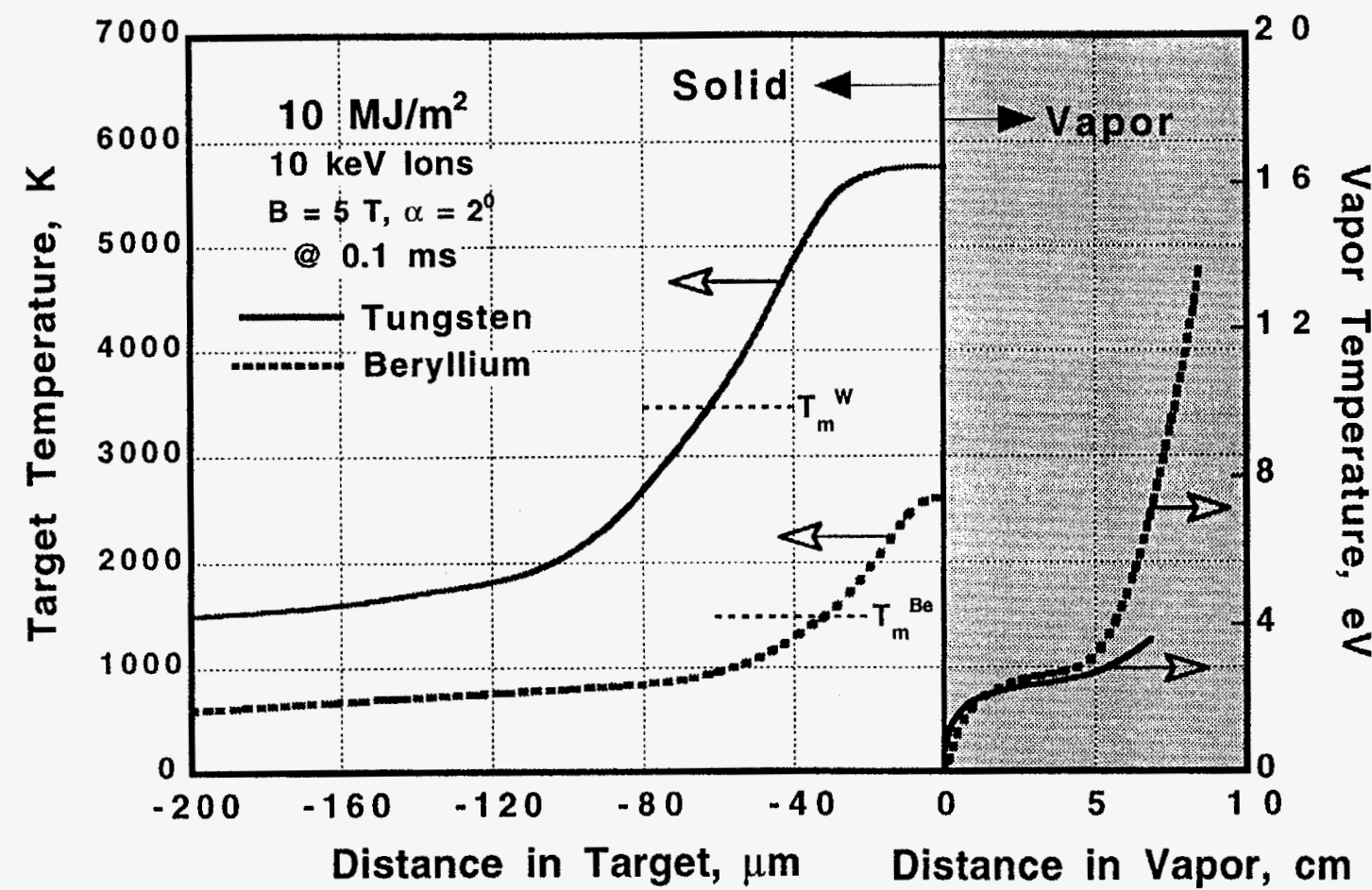

Fig. (2) 


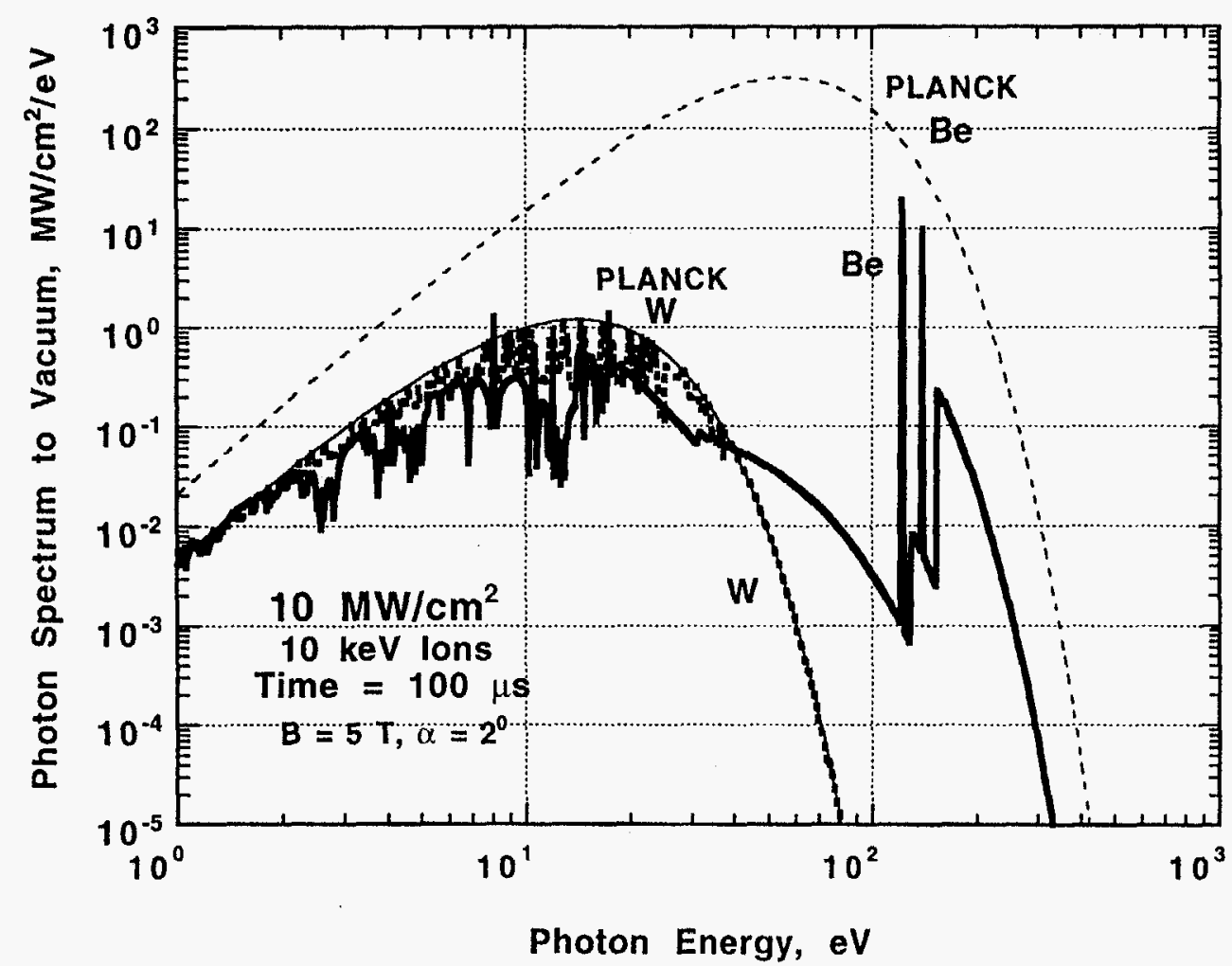

Fig.(3) 


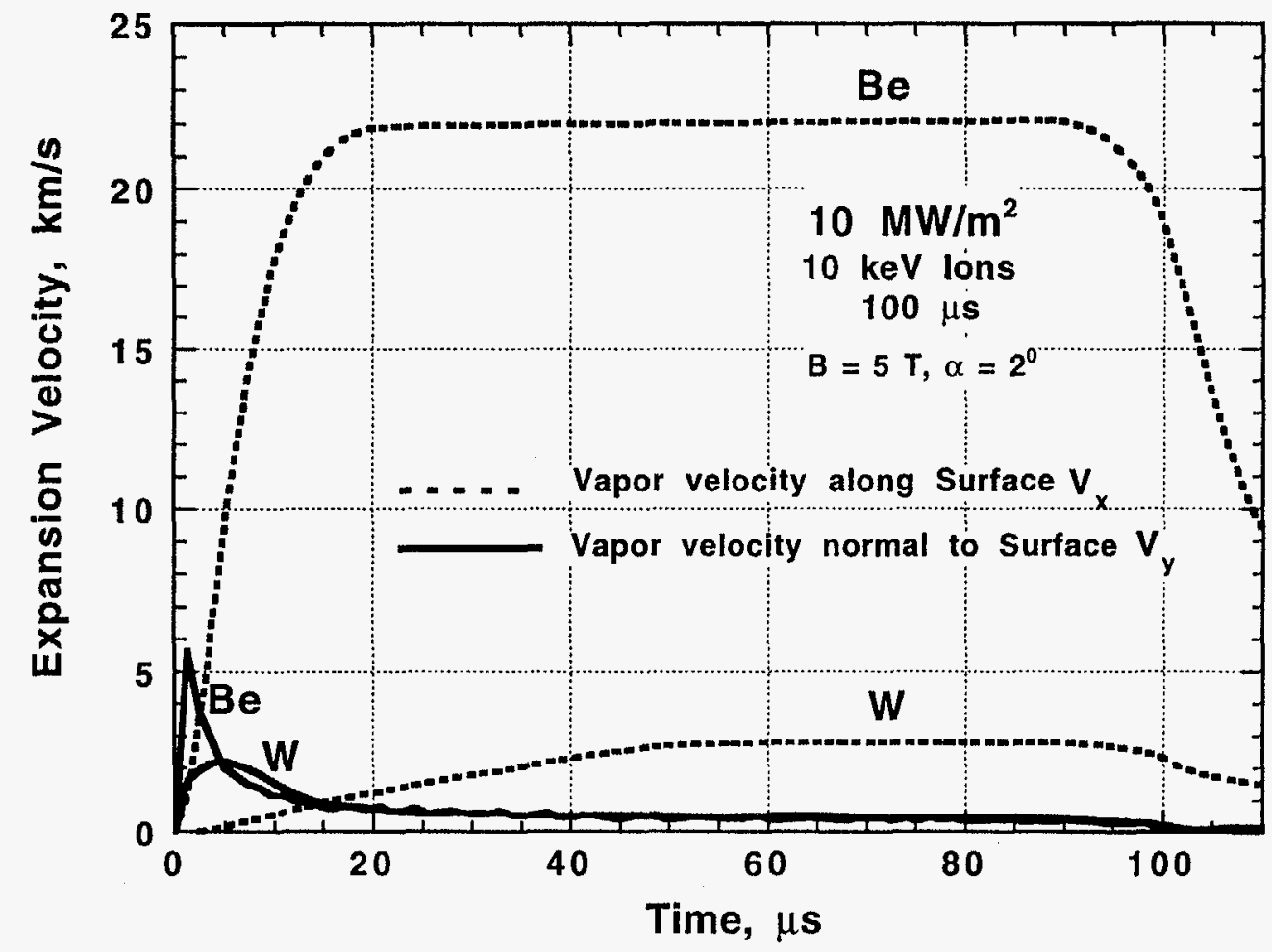

Fig. (4) 


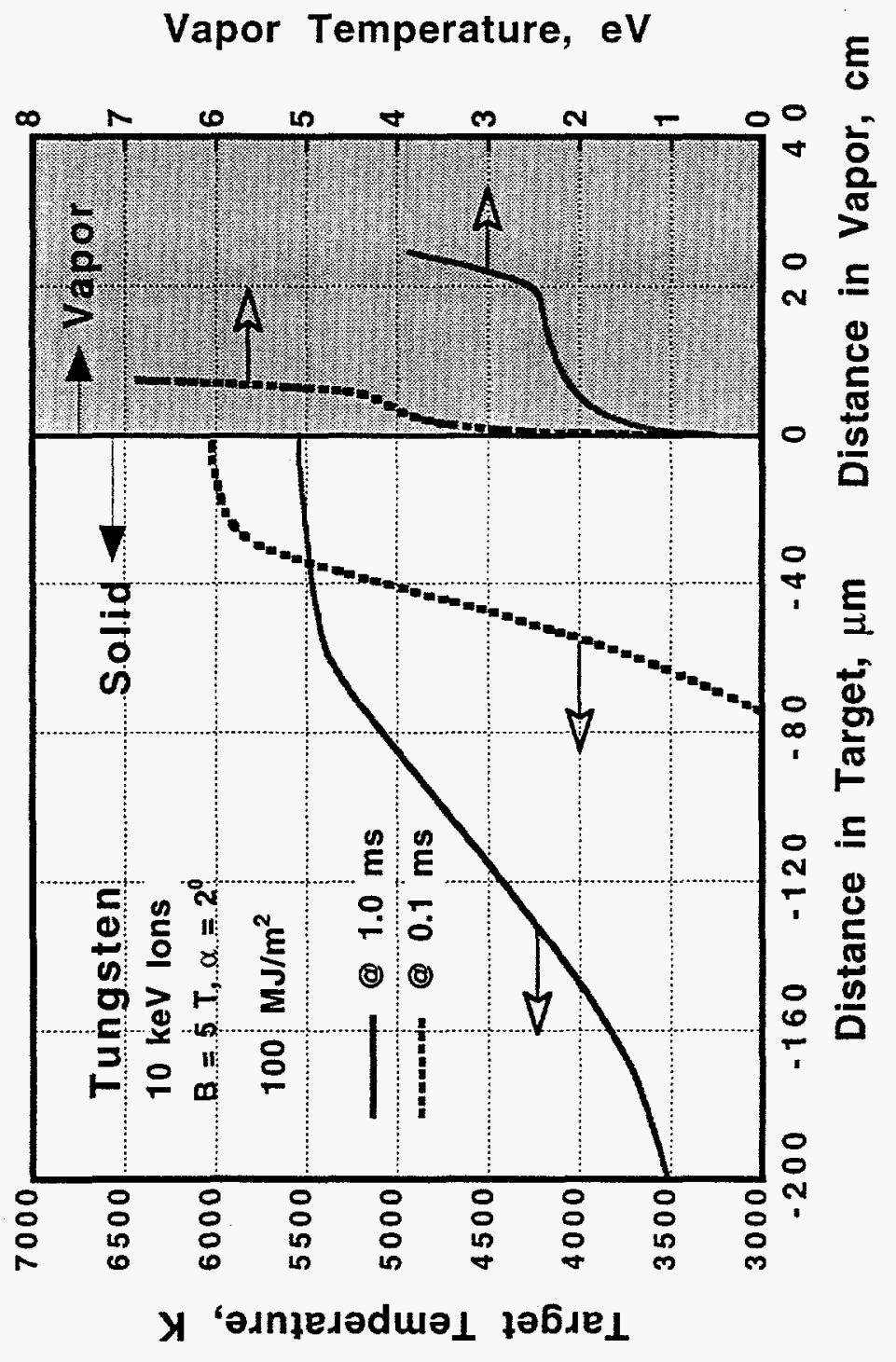

(b) 4 


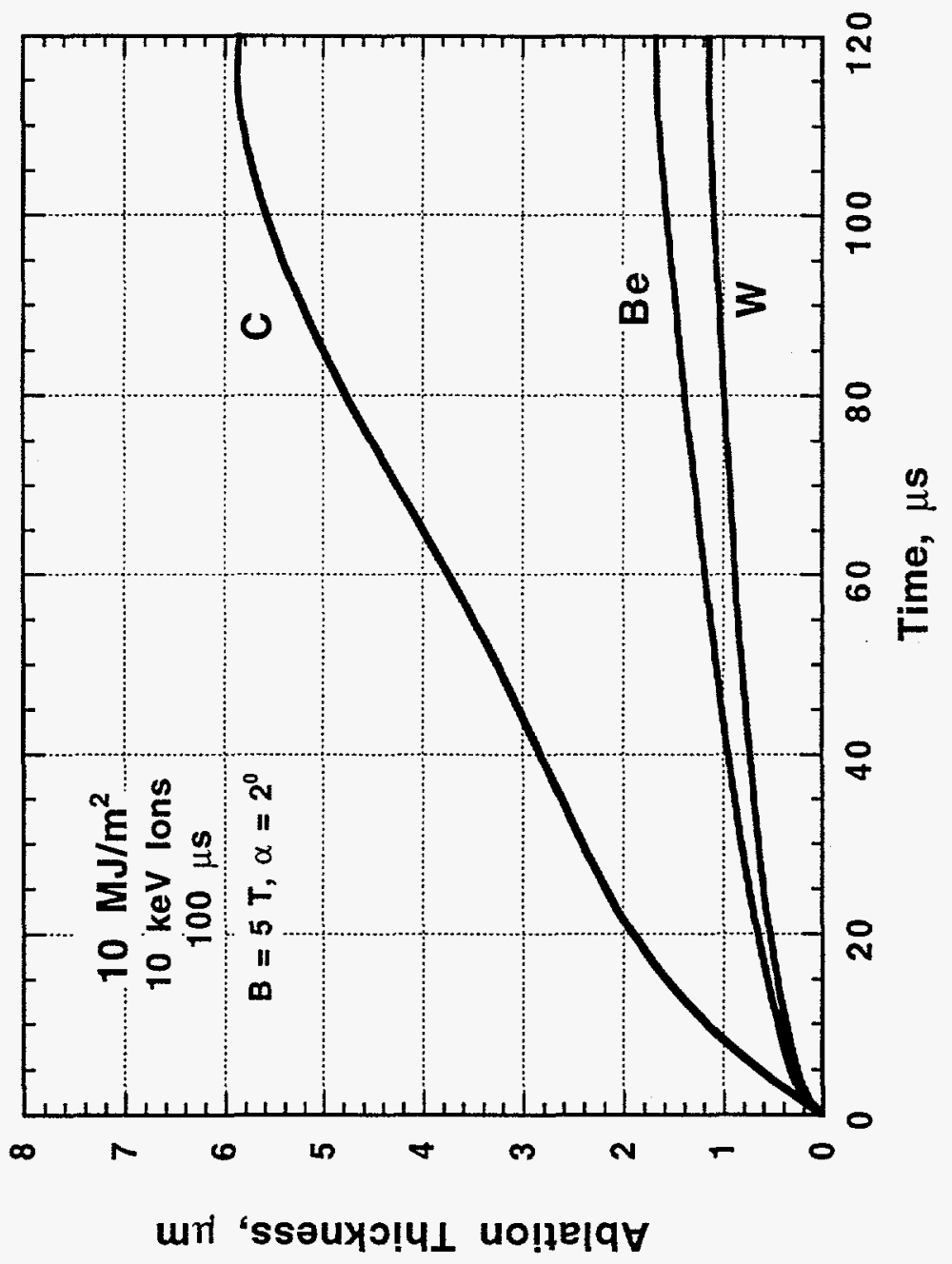

(v) 手 


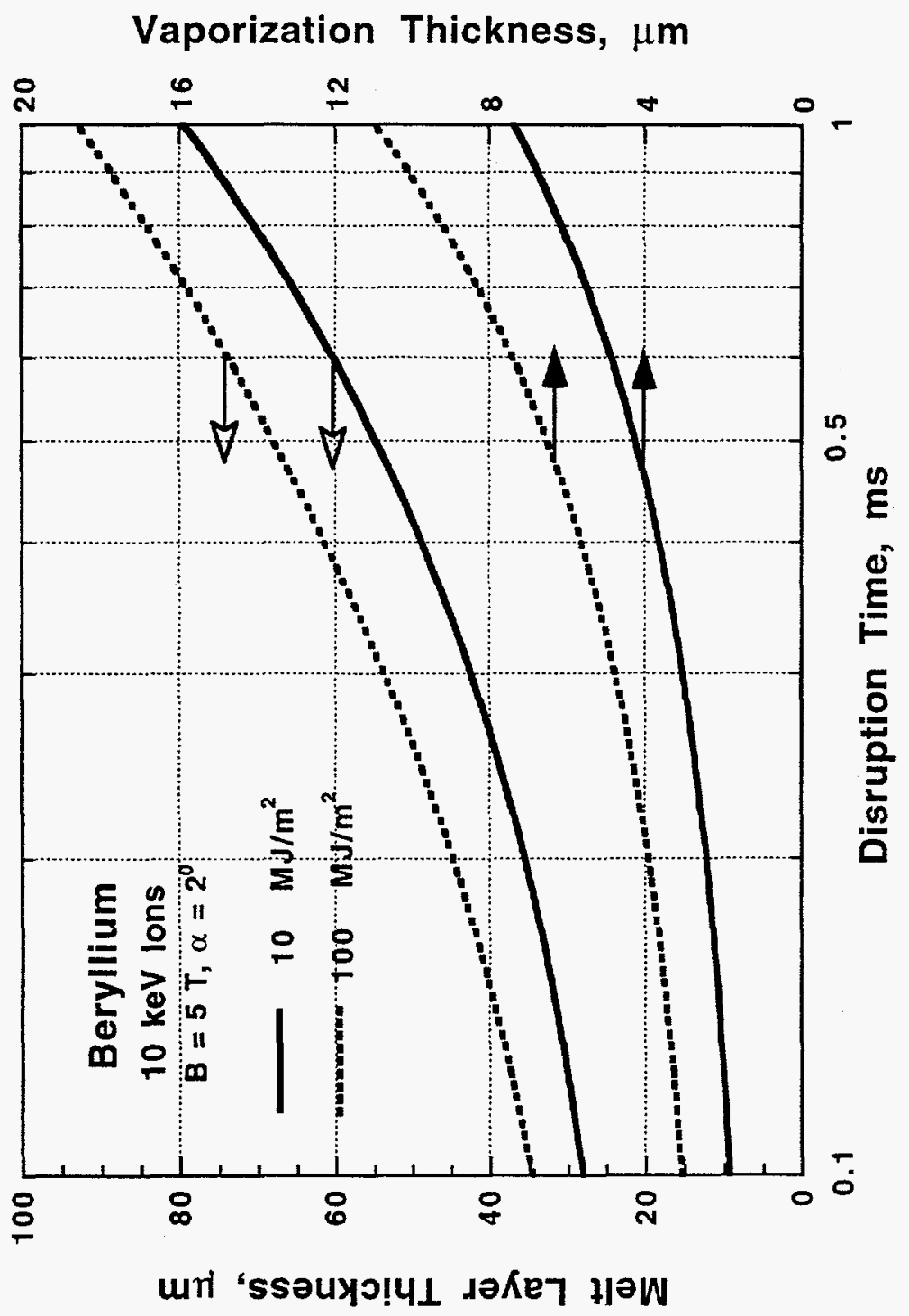

(A)

2 


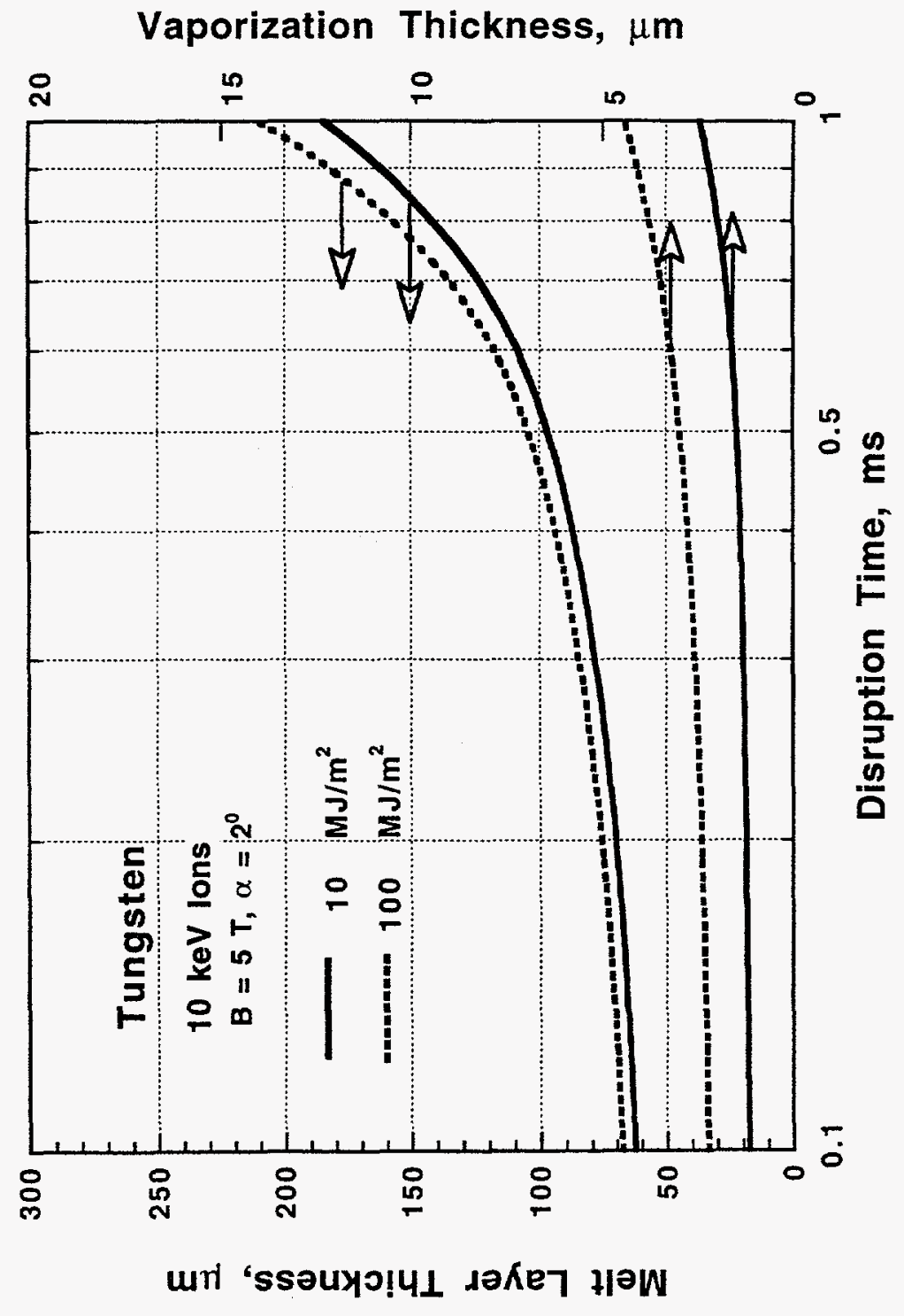

(a) i 


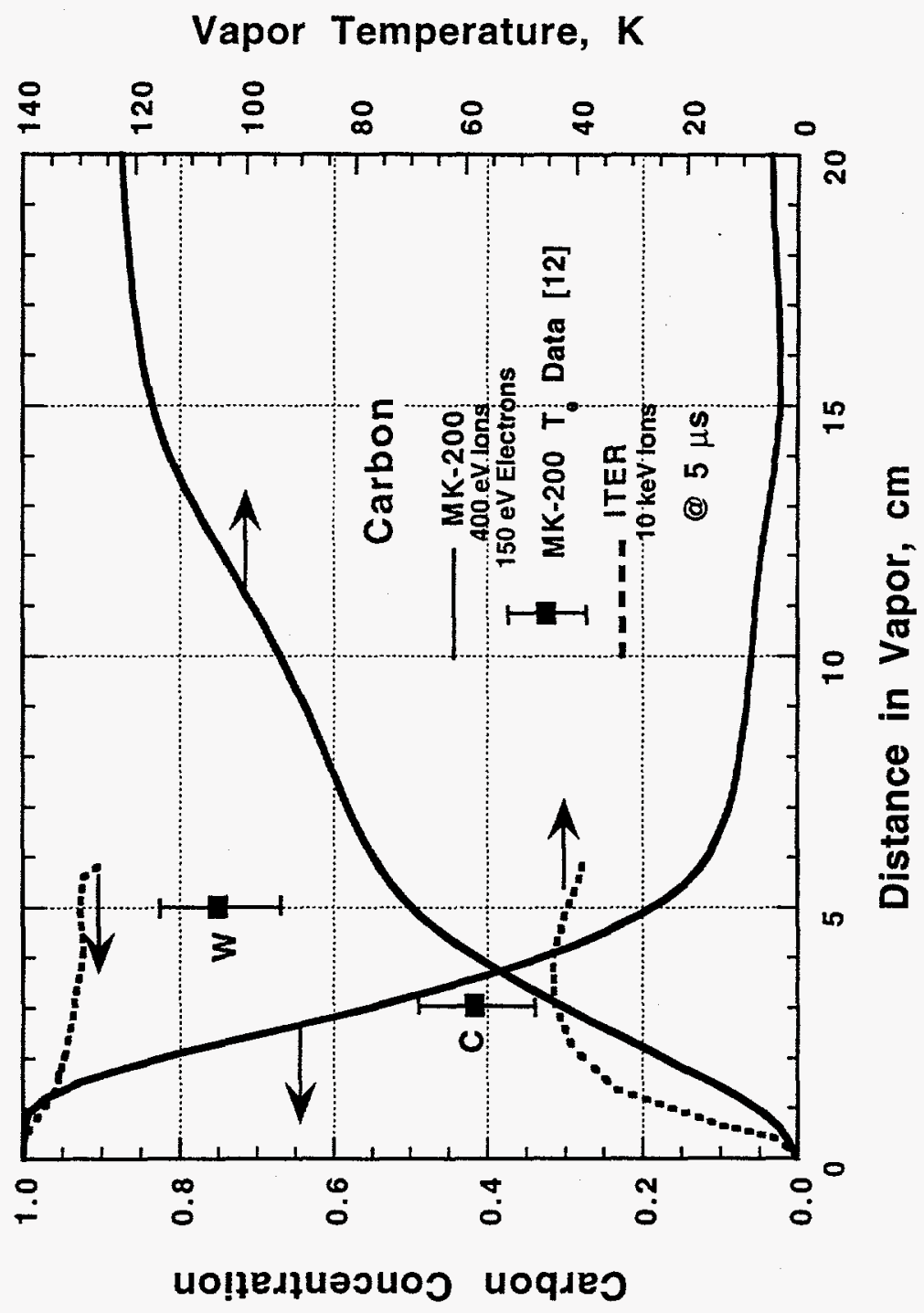

(a)

210 